\title{
Prognostic significance of vascular endothelial cell growth factors $-A,-C$ and $-D$ in breast cancer and their relationship with angio- and lymphangiogenesis
}

\author{
RAA Mohammed ',2, A Green², S El-Shikh², EC Paish², IO Ellis² and SG Martin*,I \\ 'Department of Clinical Oncology, University Hospitals, City Hospital Campus, University of Nottingham, Hucknall Road, NG5 IPB, Nottingham, UK; \\ ${ }^{2}$ Histopathology Departments, University Hospitals, City Hospital Campus, University of Nottingham, Hucknall Road, NG5 IPB, Nottingham, UK
}

Vascular endothelial cell growth factors (VEGF)-A, $-C$ and -D have potent angio and lymphangiogenic functions in experimental models, although their role in the progression of human breast cancer is unclear. The aims of the current study were to examine the relationship between the expression of the aforementioned growth factors with the angio and lymphangiogenic characteristics of breast cancer, and to assess their suitability as potential prognostic factors. Paraffin-embedded sections of 177 primary invasive breast cancer, with complete clinical follow-up information for 10 years, were stained for VEGF-A, -C, -D, podoplanin and CD34 using standard immunohistochemical approaches. The expression of the growth factors was correlated with clinicopathological criteria and patients' survival. Lymph vessel density (LVD) and microvessel density (MVD) were assessed and correlated with expression of the growth factors. Vascular endothelial cell growth factor-A, -C and -D were highly expressed in 40, 37 and $42 \%$ of specimens, respectively. High expression of VEGF-A and - C, but not of -D, was associated with a higher LVD $(P=0.013$ and $P=0.014$, respectively), a higher MVD $(P<0.00 \mathrm{I}$ and $P=0.002$, respectively), the presence of lymph node metastasis $(P<0.00 \mathrm{I}$ and $P<0.00 \mathrm{I}$, respectively), distant metastasis $(P=0.010$ and $P=0.008$, respectively) and a shorter Overall Survival $(P=0.029$ and 0.028 , respectively). In conclusion, breast cancers that express high levels of VEGF-A and $-C$ are characterised by a poor prognosis, likely through the induction of angio and lymphangiogenesis. Examination of expression of VEGF-A and -C in breast cancer may be beneficial in the identification of a subset of tumours that have a higher probability of recurrence and metastatic spread.

British Journal of Cancer (2007) 96, 1092- I 100. doi:1 0.1038/sj.bjc.6603678 www.bjcancer.com

Published online 13 March 2007

(c) 2007 Cancer Research UK

Keywords: breast cancer; vascular endothelial cell growth factors; VEGF-A; VEGF-C; VEGF-D; lymphangiogenesis; angiogenesis

Angiogenesis, the formation of new blood vessels, and lymphangiogenesis, the formation of new lymphatics, are complex processes in which different signalling systems work together; the main system being the vascular endothelial cell growth factor (VEGF) family of proteins and the associated receptors. This system is composed of a family of glycoprotein growth factors; VEGF-A, -B, -C, -D, -E and the placenta growth factor (PLGF) (Ferrara and Davis-Smyth, 1997). They bind selectively, with different affinities to three receptors that belong to the superfamily of receptor tyrosine kinases; VEGFR-1, -2 and -3. Both VEGFR-1 and VEGFR-2 are expressed on the surface of blood endothelial cells (BECs). Vascular endothelial cell growth factor receptor-3 is expressed throughout both blood and lymphatic vasculature during embryological development and in tumour tissues, however in normal adult tissues it is restricted to lymphatic endothelial cells (LECs) (Partanen and Paavonen, 2001).

Vascular endothelial cell growth factor-A, a multifunctional cytokine secreted by a large variety of cells, is known to play an

*Correspondence: Dr SG Martin;

E-mail: stewart.martin@nottingham.ac.uk

Received I5 November 2006; revised 2 February 2007; accepted 12 February 2007; published online 13 March 2007 essential role in vasculogenesis and angiogenesis (Fox et al, 2001). During transcription, it undergoes alternative splicing, yielding multiple mature glycoprotein isoforms with different amino-acid lengths (VEGF-A165, -A121, -A189 and -A206) that are capable of inducing proliferation and migration of endothelial cells (ECs) (Ferrara and Bunting, 1996). Expression of VEGF-A has been found to be upregulated and suggested to be associated with progression of certain types of human tumours such as lung (Han et al, 2001), eosophageal (Kleespies et al, 2005) and colorectal cancers (Des Guetz et al, 2006). Studies on human breast cancer reported a significant relationship between high expression of VEGF-A and tumours with higher proliferation rate, negative oestrogen receptor status (Fuckar et al, 2006) and poor prognosis (Linderholm et al, 1999).

The role of VEGF-A as a potent angiogenic factor in malignant tumours is well established, but it has long been thought that it had no influence upon lymphangiogenesis. It has recently been reported, however, that VEGF-A can induce lymphangiogenesis as well as angiogenesis (Nagy et al, 2002). The relationship between the expression of VEGF-A and lymphangiogenesis in breast cancer has not been studied and is a component of the current study.

VEGF-C and -D have potent lymphangiogenic functions through stimulation of VEGFR-3 on the surface of LECs. They are produced in pro-prepeptide dimers that undergo proteolysis 
in the extracellular matrix (ECM) to a mature form that has much higher affinity to VEGFR-3, inducing lymphangiogenesis, but can also bind VEGFR-2, inducing angiogenesis (Joukov et al, 1996). Overexpression of VEGF-C and -D in experimental tumour models has been found to be significantly associated with the formation of new lymphatics (Skobe et al, 2001; Stacker et al, 2001; Von Marschall et al, 2005), however, their effect in human cancers is a matter of controversy with some studies finding a significant association with tumour angiogenesis, lymphangiogenesis, lymph node (LN) status and prognosis (Nakamura et al, 2003a, 2005) and others no relationships (Currie et al, 2004).

The mainstay of assessment of tumour vascularity has been counting the number of immunohistochemically identified microvessels in vascular hot spots, known as the microvessel density (MVD) (Weidner et al, 1992), using vascular markers such as factor VIII-related antigen, CD34 and CD31, with a recent review recommending procedures that should be followed for the assessment of MVD in breast cancer (Fox and Harris, 2004). The assessment of lymphatic characteristics in malignant tumours has historically been difficult owing to the lack of availability of lymphatic-specific markers. Such markers have recently been characterised and become commercially available. Markers include podoplanin, D2-40, LYVE-1 and Prox-1. The count of positively stained vessels per tumour area, lymph vessel density (LVD), has been used to assess lymphangiogenic characteristics in tumour specimens (Rodriguez-Niedenfuhr et al, 2001; Sahni et al, 2005). High MVD (Weidner et al, 1991; Bevilacqua et al, 1995; Toi et al, 1995b; Choi et al, 2005) and high LVD (Choi et al, 2005) in breast cancer have been reported to be associated with more aggressive tumour behaviour and poorer survival.

Although a number of studies have examined the relationship between the expression of each of the VEGFs with patient prognosis, angiogenic and lymphangiogenic characteristics in breast cancer, examination of the expression of all three, together, in a well-characterised series of breast cancers with long-term follow-up has not been conducted. The aims of the current study were to: (a) investigate the expression of VEGF-A, -C and -D in human breast cancer via immunohistochemistry, (b) examine the tumour lymphatic and vascular characteristics by counting lymph vessels/area to determine LVD and counting blood vessels via the Chalkley method to assess MVD and (c) conduct correlations between expression of the growth factors in relation to patient clinicopathological data, survival, lymphangiogenesis and angiogenesis to examine the role that they play in the regulation of such processes and in the progression of breast cancer.

\section{MATERIALS AND METHODS}

\section{Patients and tissue samples}

One hundred and seventy-seven paraffin-embedded archival specimens of primary invasive breast cancer were retrieved from the Department of Histopathology, Nottingham University Hospitals, City Hospital Campus. The median age of patients at time of diagnosis was 57 years (range 32-70 years). Fifty-one patients $(28.2 \%)$ were younger than 50 years. Sixty-five $(36.7 \%)$ of the specimens were $<1.5 \mathrm{~cm}$. At the time of primary diagnosis, 52 (29.4\%) patients had positive LNs. Most of the tumours were stage I $(n=121,68.4 \%)$ and stage II $(n=43,24.3 \%)$ disease. Complete clinical follow-up information was available for all 177 patients, with ethical approval obtained for analysis from Nottingham Local Research Ethics Committee (REC C2020313). The median followup period was 96 months. Forty-one patients developed regional recurrence by the time of the last follow-up and 16 patients died from breast cancer.

\section{Immunohistochemistry}

Staining with two lymphatic markers, D2-40 (SIGNET, 730-16) and podoplanin was carried out on paraffin-embedded blocks of tonsil, LN and breast cancer tissues. Recently, however, Schacht et al (2005) found that D2-40 and podoplanin detect the same protein on LECs. As both markers showed the same intensity of staining, and the same pattern, either can be used to generate the same results.

A representative, paraffin-embedded section from each specimen was stained with Podoplanin (polyclonal Ab, AngioBio, CA, USA, 11-003, 1:100 dilution), CD34 (polyclonal Ab, SEROTEC, Oxford, UK, MCAP547, 1:500 dilution), VEGF-A (monoclonal Ab, LAB VISION, CA, USA, RM-9128-S, $1: 100$ dilution), VEGF-C (polyclonal Ab, ZYMED, CA, USA, 18-2255, 1:75 dilution) and VEGF-D (monoclonal Ab, R\&D, Abingdon, UK, MAB286 in $1: 400$ dilution). Briefly, 4- $\mu \mathrm{m}$-thick sections were deparaffinised with xylene and rehydrated. Antigen retrieval for CD34, VEGF-A, -C and $-\mathrm{D}$ was achieved by incubating sections in $0.01 \mathrm{moll}^{-1}$ sodium citrate buffer ( $\mathrm{pH} 6.0)$ in a $800 \mathrm{~W}$ microwave for $20 \mathrm{~min}$; $10 \mathrm{~min}$ at $800 \mathrm{~W}$ then $10 \mathrm{~min}$ at $100 \mathrm{~W}$. Podoplanin did not require antigen retrieval. Endogenous hydrogen peroxidase $\left(\mathrm{H}_{2} \mathrm{O}_{2}\right)$ reactivity was blocked with $3 \% \mathrm{H}_{2} \mathrm{O}_{2}$. Nonspecific reactions were blocked by use of normal swine serum for $20 \mathrm{~min}$. Sections were incubated for $1 \mathrm{~h}$ at room temperature for all primary antibodies except for VEGF-D that was incubated overnight at $4{ }^{\circ} \mathrm{C}$. After washing, sections were treated with commercial biotinylated secondary anti-immunoglobulin, followed by avidin coupled to biotinylated horseradish peroxidase, at room temperature, according to the manufacturer's instructions using streptABC kit (StreptABComplex/HRP Duet, Mouse/Rabbit kit, DAKO Corporation, Denmark, K0492). Immunohistochemical reactions were developed with $3,3^{\prime}$ diaminobenzidine as the chromogenic peroxidase substrate (DAKO, K3468). Sections were then counterstained with Myer's haematoxylein, dehydrated, fixed in xylene and mounted with DPX. Sections from placenta were used as a positive control, for VEGFs and CD34, and sections from LN were used for podoplanin. For negative controls, sections were stained using the same procedure after omitting primary antibody.

\section{Evaluation of growth factor expression}

Expression of VEGF-A, -C and -D was assessed semiquantitatively using an immunohistochemical score ( $H$ score). Staining intensity was given four grades: none (0), weak (1), moderate (2) and strong (3). $H$ score was calculated by multiplying the percentage of positive carcinoma cells by the staining intensity, this gave an $H$ score ranging from 0 to 300 . The median of the score was selected as the cutoff level according to which tumours were categorised into low- and high-expressing tumours. Median values were 160 for VEGF-A, 140 for VEGF-C and 130 for VEGF-D. This method, in selecting an appropriate cutoff level, has been used in previous studies (Yang et al, 2002).

\section{Assessment of LVD}

The commonly used method for assessment of density of lymph vessels in tumour has been counting the number of immunohistochemically identified lymphatics in the most vascularised tumour areas; hot spots (Giorgadze et al, 2004; Koskinen et al, 2005; Nakamura et al, 2005). In the present study, a modified procedure was used, where LVD was assessed by counting all lymph vessels in the whole tumour section, using $\times 100$ magnification with a surface area of $3.46 \mathrm{~mm}^{2}$. Although easier to use the $\times 200$ magnification to assess LVD, the strong staining of lymphatics allowed easy recognition of vessels at $\times 100$, especially when examining the whole tumour section. The sum of lymph vessels was divided by the sum of the surface area of all 
counted fields to adjust LVD per $\mathrm{mm}^{2}$. Lymph vessel density was presented as number of lymph vessels per $\mathrm{mm}^{2}$. Although more labour intensive, such methodology allows a more accurate assessment of lymphatic density. The distribution of lymphatics and prevalence of lymphovascular invasion will be the focus of a separate report.

\section{Assessment of MVD}

Sections stained with CD34 were used for the evaluation of MVD using the Chalkley counting method. Each section was first scanned at low-power magnification $(\times 40)$ to select the most vascularised areas; three hot spots were selected. Two authors first examined $10 \%$ of specimens to agree on which fields to be used as hot spots. A 25-point Chalkley eyepiece graticule was applied to each hot spot and oriented to permit the maximum number of points to hit on, or within the areas of immunohistochemically highlighted microvessel using $\times 200$ magnification. A Chalkley count for an individual tumour was taken as the mean value of the three graticule counts (Fox and Harris, 2004).

For assessment of LVD, MVD and expression of VEGFs, a second investigator also blinded to the patients' clinical characteristics and survival data, independently assessed $20 \%$ of sections. A $\kappa>0.90$ was obtained, indicating a very good correlation between observers.

\section{Statistical analysis}

Four levels of statistics were performed using SPSS for windows, version 13: (1) Mann-Whitney tests were conducted to compare means of LVD and MVD (as a continuous data) between different clinicopathological groups and (2) specimens were divided into two categories according to the median values of LVD and MVD. The association between expression of VEGFs and LVD, MVD and clinicopathological criteria was evaluated in univariate analysis using a $2 \times 2$ table and $\chi^{2}$ test, (3) survival analysis of disease-free interval (DFI) and overall survival (OS) was accomplished using the Kaplan-Meier method and the statistical significance of differences in the cumulative survival curves between groups was evaluated by the long-rank test. Multivariate survival analysis was performed using the Cox's proportional hazard method and (4) the Kappa $(\kappa)$ agreement test was used to assess agreement between observers. All statistical analyses were two sided with significance defined as $P<0.05$.

\section{RESULTS}

\section{Expression of VEGFs}

Expression of all three VEGFs showed a positive cytoplasmic staining in the breast cancer cells, with granular and heterogeneous staining in some specimens. Positive staining for VEGF-A was detected in the normal mammary epithelial cells adjacent to tumour, in the ECs and in the tumour-associated macrophages (Figure 1A). Seventy-seven (40\%) specimens of breast carcinomas showed high expression of VEGF-A, with stronger staining intensity found in the invasive component of the tumour and vascular emboli. In many specimens, the staining intensity was heterogeneous among tumour areas (Figure 1B). Consistent with the findings of others (Nakamura et al, 2003a; Jennbacken et al, 2005), positive staining with VEGF-C and -D was observed in the normal mammary epithelial cells, tumour-associated macrophages, stromal cells and ECs (Figure 1C)Vascular endothelial cell growth factor-D showed positive staining in the vascular smooth muscle fibres. Sixty-seven (37\%) and 91 (44\%) specimens showed strong staining with VEGF-C and -D, respectively (Figures 1C and D).

The relationship between expressions of the three growth factors was analyzed. Specimens with high expression of VEGF-A found to be significantly associated with high expression of VEGF-C
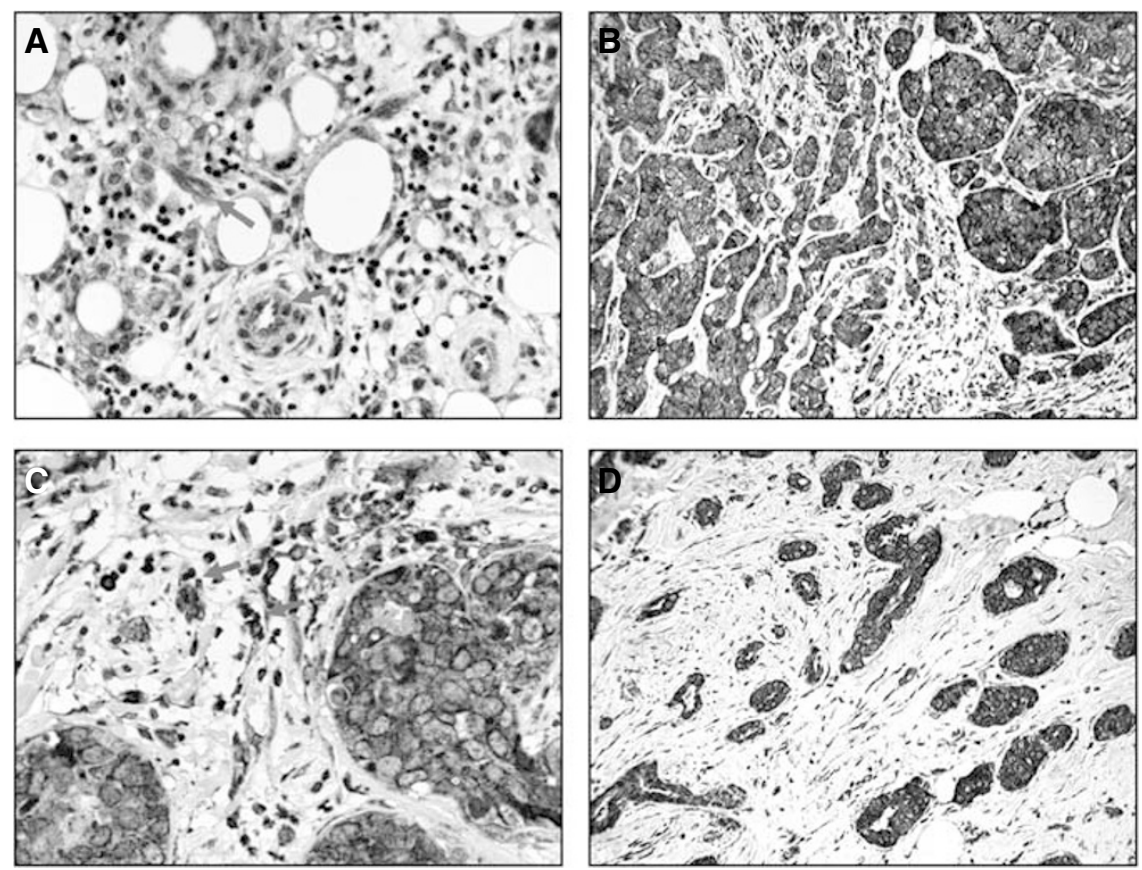

Figure I (A) Peritumoural area of a breast carcinoma section stained with VEGF-A showing positive staining in the endothelial cells (red arrow) and in the macrophages (green arrow), $\times 400$. (B) Grade-III infiltrating breast carcinoma showing heterogeneous staining intensity for VEGF-A in the same specimen; tumour sheets with weak staining intensity on the left and others with strong staining intensity on the right, $\times 200$. (C) VEGF-C-stained tumour section showing strong positive staining in the tumour-associated macrophages (red arrow) and in the tumour cells, $\times 400$. (D) Grade-ll infiltrating breast carcinoma showing strong positive staining with VEGF-D, $\times 100$. 
$(P=0.046)$ but not with expression of VEGF-D $(P=0.354)$. There was, however, a significant association between expression of VEGF-C and -D where $64 \%$ of cases with high expression of VEGF-C had high expression of VEGF-D $(P=0.001)$, Table 1.

\section{Relationships amongst expression of VEGFs, angiogenesis and lymphangiogenesis}

Lymph vessel density ranged from $0.015 \mathrm{~mm}^{-2}$ to $8.59 \mathrm{~mm}^{-2}$ with a mean of $1.7 \mathrm{~mm}^{-2} \pm 0.1$ and a median of 1.37 . Fifty-five $(31.1 \%)$ specimens were characterised with a high LVD, Figure 2A. MVD ranged from 1.0 to 7.6 with a mean of $2.5 \pm 0.09$ and a median of 2.3. Seventy-six (42.9\%) specimens were characterised with high MVD, Figure 2B. The relationship amongst VEGF-A, $-\mathrm{C}$ and $-\mathrm{D}$ expression, MVD and LVD are summarised in Table 2. Tumours with high expression of VEGF-A were significantly associated with both a higher MVD $(P<0.001)$ and higher LVD $(P=0.013)$. Such tumours had a mean MVD of $2.9 \pm 0.15$ and a mean LVD of $1.5 \pm 0.11$ compared to $2.2 \pm 0.11$ and $2.0 \pm 0.19$ for specimens with low VEGF-A expression respectively. Similar findings were found with tumours with high expression, of VEGF-C. Such tumours had a mean MVD of $2.8 \pm 0.17$ and a mean LVD of $2.2 \pm 0.22$ compared with $2.3 \pm 0.11$ and $1.5 \pm 0.11$ for patients with low VEGF-C expression respectively $(P=0.002$ for MVD and $P=0.014$ for LVD), such relationships are illustrated using box plots in Figure 3. No statistical significant association was found between expression of VEGF-D and LVD or with MVD.

\section{Relationships between expression of VEGFs and clinicopathological criteria}

A comparison between low and high growth factor expressing tumours was conducted to examine for potential associations with

Table I Relationship between expression of VEGF-A, VEGF-C and VEGF-D

\begin{tabular}{|c|c|c|c|c|c|c|}
\hline & \multicolumn{3}{|c|}{ VEGF-C no. (\%) } & \multicolumn{3}{|c|}{ VEGF-D no. (\%) } \\
\hline & Low & High & $P$-value & Low & High & $P$-value \\
\hline \multicolumn{7}{|l|}{ VEGF-A } \\
\hline Low & 75 (7I) & $31(28)$ & & 61 (57) & $45(42)$ & \\
\hline High & $39(55)$ & $32(45)$ & 0.046 & $35(49)$ & $36(5 \mathrm{I})$ & 0.354 \\
\hline \multicolumn{7}{|l|}{ VEGF-C } \\
\hline Low & & & & $73(64)$ & $41(36)$ & \\
\hline High & & & & $23(36)$ & $40(64)$ & 0.001 \\
\hline
\end{tabular}

VEGF-A = vascular endothelial cell growth factor-A; VEGF-C = vascular endothelial cell growth factor-C. Bold values denotes statistical significance. clinicopathological characteristics (Table 2). High expression of VEGF-A was significantly associated with tumours larger than $1.5 \mathrm{~cm}(P=0.038)$ and also with grade III tumours $(P<0.001)$. Sixty-four percent of breast carcinomas with negative oestrogen receptors (ER) and 55\% of those with negative progesterone receptor (PR) status express significantly higher levels of VEGF-A than hormonal receptor positive carcinomas $(P<0.001$ and $P=0.004$, respectively). A significant association was also found with the presence of axillary LN $(P<0.001)$ and presence of distant metastasises $(P=0.010)$ but not with patients' age.

A significant positive relationship between VEGF-C expression and presence of LN metastasis $(P<0.001)$ was detected. Tumours larger than $1.5 \mathrm{~cm}$ had a significantly higher level of VEGF-C expression $(P=0.023)$ but unlike VEGF-A, no association was found in relation to the tumour grade $(P=0.214)$ or with the hormonal receptor status $(P=0.865$ for $\mathrm{ER}$ and $P=0.624$ for PR).

No significant association was found between expression of VEGF-D and any of the clinicopathological criteria. However, a larger proportion of tumours with high expression with VEGF-D $(54 \%)$ had LN metastasis compared to low expressing tumours (42\%) but was not statistically significant $(P=0.187)$.

\section{Prognostic significance of growth factor expression}

Kaplan-Meier analysis for OS and DFI were conducted to investigate whether expression of VEGF-A, -C and -D had any prognostic significance. From univariate analysis, high expression of VEGF-A was significantly associated with shorter OS $(P=0.029)$, Figure 4A. High expression of VEGF-C was significantly associated with both shorter OS $(P=0.025$ and DFI $(P=0.028)$ (Figure $4 \mathrm{~B}$ and $\mathrm{C})$, however VEGF-D was neither associated with DFI nor OS. Only VEGF-C retained significance upon multivariate analysis, when adjusted for tumour size, tumour grade and patient age $(P=0.047$, hazard ratio $2.854 ; 95 \%$ confidence interval 1.016-8.015).

Tumours were subsequently recategorised according to expression of VEGF-A and -C into four groups; group (A); tumours with low expression of both VEGF-A and -C, group (B); tumours with high expression of VEGF-A, group (C); tumours with high expression of VEGF-C and group (D); tumours with high expression of both VEGF-A and -C. On survival analysis, tumours with high expression of both VEGF-A and $-\mathrm{C}$ had the shortest OS when compared with the other three groups $(P<0.001)$ (Figure 4D). The prognostic significance of LVD and MVD will be a focus of a separate report examining the role of lymphatic distribution, density and lymphovascular invasion in relationship to clinicopathological criteria of tumours and patients' survival.
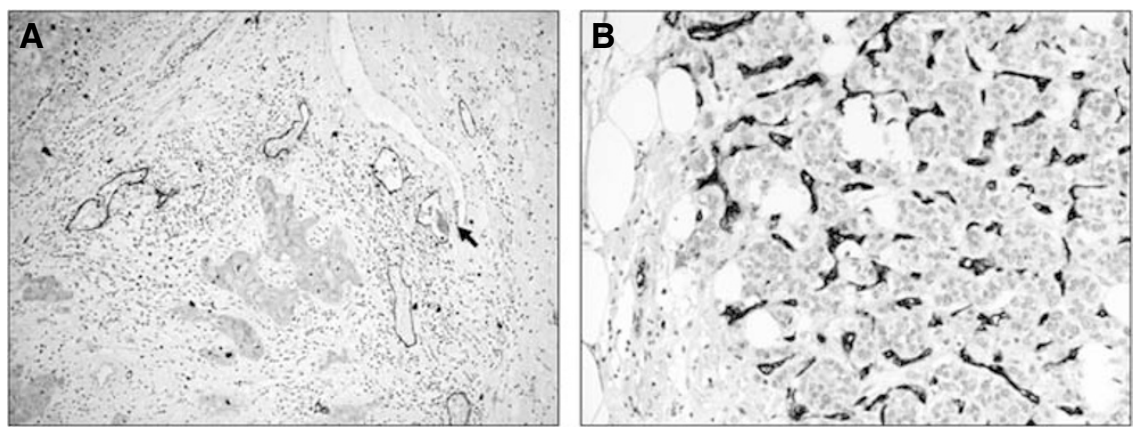

Figure 2 (A) Infiltrating duct carcinoma of the breast stained with podoplanin showing multiple lymphatics at the peritumoural area with a lymphatic invasion in one of the vessels (arrow), $\times 200$. (B) Infiltrating duct carcinoma of the breast stained with CD34 showing high MVD, $\times 200$. 
Table 2 Association between expression of VEGFs with clinicopathological criteria, lymphangiogenesis and angiogenesis

\begin{tabular}{|c|c|c|c|c|c|c|c|}
\hline & $\begin{array}{c}\text { No. of } \\
\text { patients }\end{array}$ & $\begin{array}{l}\text { No. (\%) of VEGF-A high- } \\
\text { expressing specimens }\end{array}$ & $P$-value & $\begin{array}{l}\text { No. (\%) of VEGF-C high- } \\
\text { expressing specimens }\end{array}$ & $P$-value & $\begin{array}{l}\text { No. (\%) of VEGF-D high- } \\
\text { expressing specimens }\end{array}$ & $P$-value \\
\hline \multicolumn{8}{|l|}{ Age } \\
\hline \multicolumn{8}{|l|}{ Size } \\
\hline$<1.5 \mathrm{~cm}$ & 65 & $19(29)$ & & $16(25)$ & & $28(43)$ & \\
\hline$>1.5 \mathrm{~cm}$ & 112 & $52(46)$ & 0.038 & $47(42)$ & 0.023 & $53(47)$ & NS \\
\hline$\|$ & 62 & $19(31)$ & & $29(47)$ & & $30(48)$ & \\
\hline III & 70 & $43(60)$ & $<0.001$ & $25(36)$ & NS & $29(4 I)$ & NS \\
\hline \multicolumn{8}{|l|}{ LN status } \\
\hline Negative & 125 & $36(29)$ & & $31(25)$ & & $53(42)$ & \\
\hline Positive & 52 & 35 (76) & $<\mathbf{0 . 0 0 1}$ & $32(61)$ & $<\mathbf{0 . 0 0 1}$ & $28(54)$ & NS \\
\hline \multicolumn{8}{|l|}{ NPI } \\
\hline Good & 74 & $16(22)$ & & $19(26)$ & & $33(45)$ & \\
\hline \multicolumn{8}{|l|}{$P R$} \\
\hline Negative & 72 & $4 \mid(55)$ & & $27(38)$ & & $32(44)$ & \\
\hline Positive & 93 & $30(32)$ & 0.004 & $31(33)$ & NS & $42(45)$ & NS \\
\hline \multicolumn{8}{|l|}{$R R$} \\
\hline No & 136 & $55(40)$ & & $42(31)$ & & $59(43)$ & \\
\hline Definite & 41 & $16(38)$ & NS & $21(51)$ & 0.025 & $22(54)$ & NS \\
\hline \multicolumn{8}{|l|}{ MVD } \\
\hline Low & 101 & $29(29)$ & & $31(31)$ & & $48(48)$ & \\
\hline High & 76 & $42(55)$ & $<0.001 *$ & $32(4 I)$ & $0.002 *$ & $33(43)$ & NS* \\
\hline \multicolumn{8}{|l|}{ LVD } \\
\hline Low & 122 & $42(35)$ & & $36(29)$ & & $56(46)$ & \\
\hline High & 55 & $29(5 \mathrm{I})$ & $0.013 *$ & $27(49)$ & $0.014 *$ & $25(46)$ & NS* \\
\hline \multicolumn{8}{|c|}{ LVI } \\
\hline
\end{tabular}

$\mathrm{DM}=$ distant metastasis; $\mathrm{ER}=$ oestrogen receptor; $\mathrm{LN}=$ lymph node; $\mathrm{LVD}=$ lymph vessel density; $\mathrm{MVD}=$ microvessel density; $\mathrm{NPI}=\mathrm{Nottingham}$ prognostic index; $\mathrm{NS}=$ nonsignificant; $\mathrm{PR}=$ progesterone receptor, $\mathrm{RR}=$ regional recurrence. Statistical significance was tested using $2 \times 2$ table and $\chi^{2}$ test, except $(*)$, where it was performed using Mann-Whitney test. Bold values denotes statistical significance.

\section{DISCUSSION}

The present report studied the expression of VEGF-A, -C and -D in breast cancer and correlated results with the lymphangiogenic and angiogenic characteristics of the tumours.

Vascular endothelial growth factor-A was originally identified as an EC-specific growth factor that induces angiogenesis and increases vascular permeability. It was found to be expressed in normal human tissues including liver, kidney, adrenal glands, lung and stomach (Ferrara and Davis-Smyth, 1997). In the study of Brown et al, (1993), VEGF-A mRNA was detected in the breast cancer cells whereas the corresponding proteins were found in both tumour cells and the ECs, indicating that VEGF-A is secreted by tumour cells then undergoes processing in the ECM to be entrapped by the receptors on the surface of the ECs. In the current report, and in agreement with such previous findings, positive staining for VEGF-A was detected in the ECs, in the normal epithelial mammary duct cells as well as in the tumour cells.

Similar to findings reported by other studies on breast cancer (Toi et al, 1995a; Hicklin and Ellis, 2005) and on other tumour types (Han et al, 2001), tumours with high expression of VEGF-A were characterised by a significantly higher MVD. This may be due to the multiple effects of VEGF-A on ECs. Not only is it a potent mitogenic factor but it also stimulates ECs to secrete cytokines essential for cell migration and sprouting of new vessels. It has long been supposed that VEGF-A had no association with lymphangiogenesis, however, in two recent experimental studies, VEGF-A overexpression was found to induce formation of new lymph vessels and dilatation of pre-existing ones (Nagy et al, 2002; Kunstfeld et al, 2004). This action was found to be mediated through stimulation of VEGFR-2, $\alpha 1 \beta 1$ and $\alpha 2 \beta 1$ integrins on the surface of LECs (Hong et al, 2004). Certain studies have reported a significant relationship between VEGF-A expression and lymphangiogenesis in malignant lymphoma (Kadowaki et al, 2005) and in lung carcinoma (Niki et al, 2000). The current study is the first, to our knowledge, to report such a relationship in human breast cancer, in that a significant association between VEGF-A expression and high LVD was observed. Such results suggest an important role for VEGF-A in the induction of both angiogenesis and lymphangiogenesis in breast cancer.

High expression of VEGF-A was found to be associated with tumours larger than $1.5 \mathrm{~cm}$ in size. This is in agreement with a previous study on breast cancer (Toi et al, 1995a) and one on lung cancer (Niki et al, 2000), where they found that high expression of VEGF-A was not only associated with larger tumours but also with 


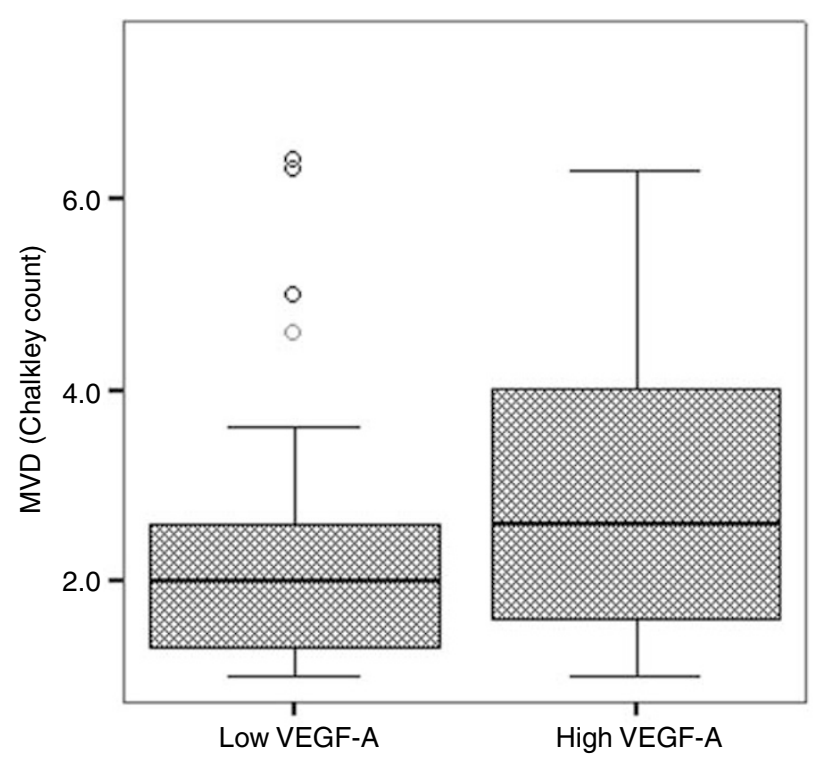

Level of VEGF-A expression

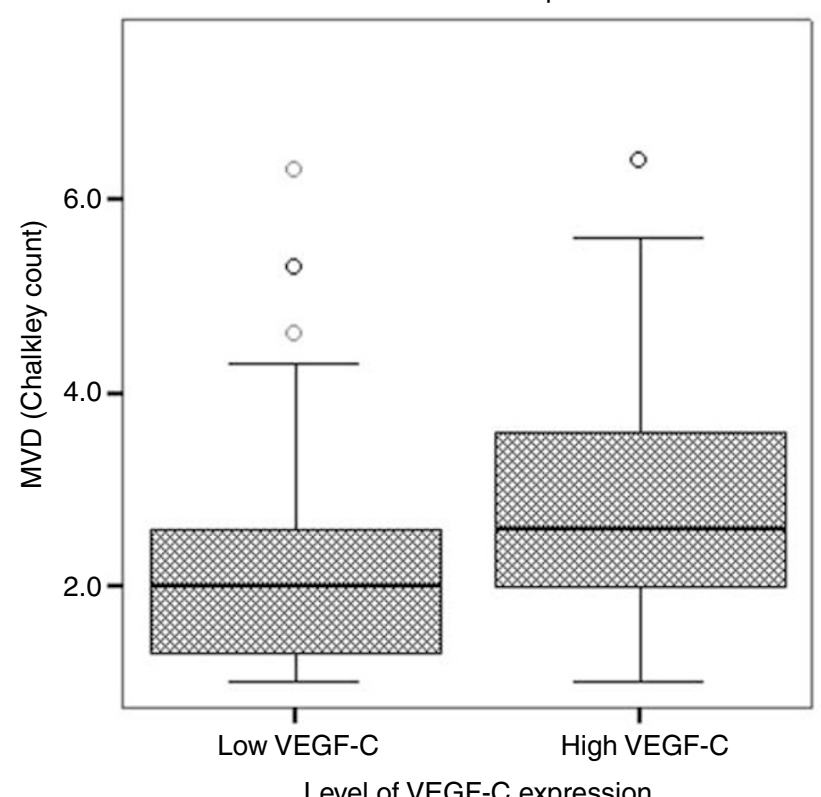

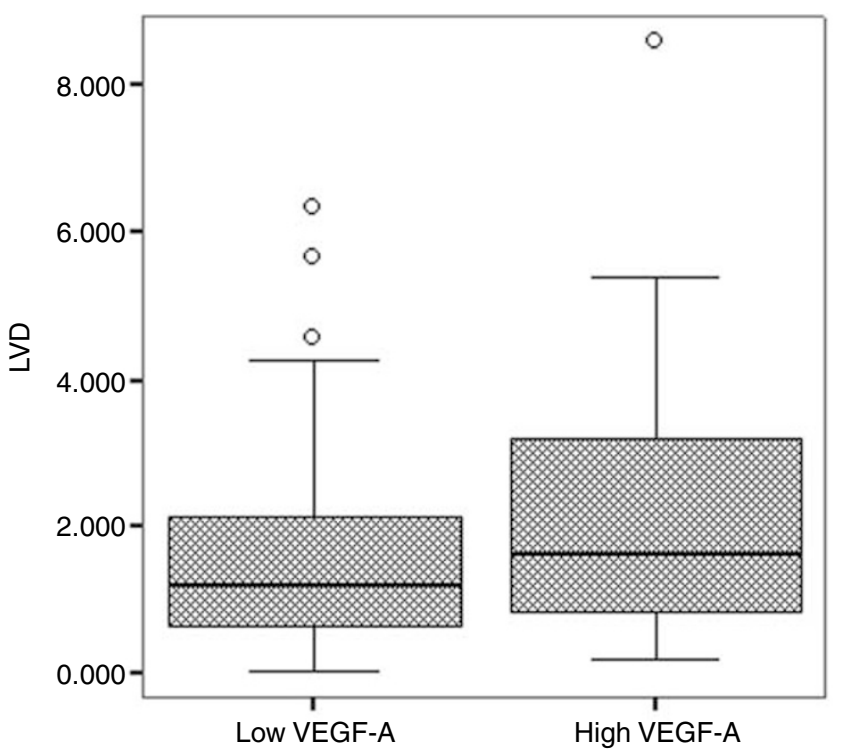

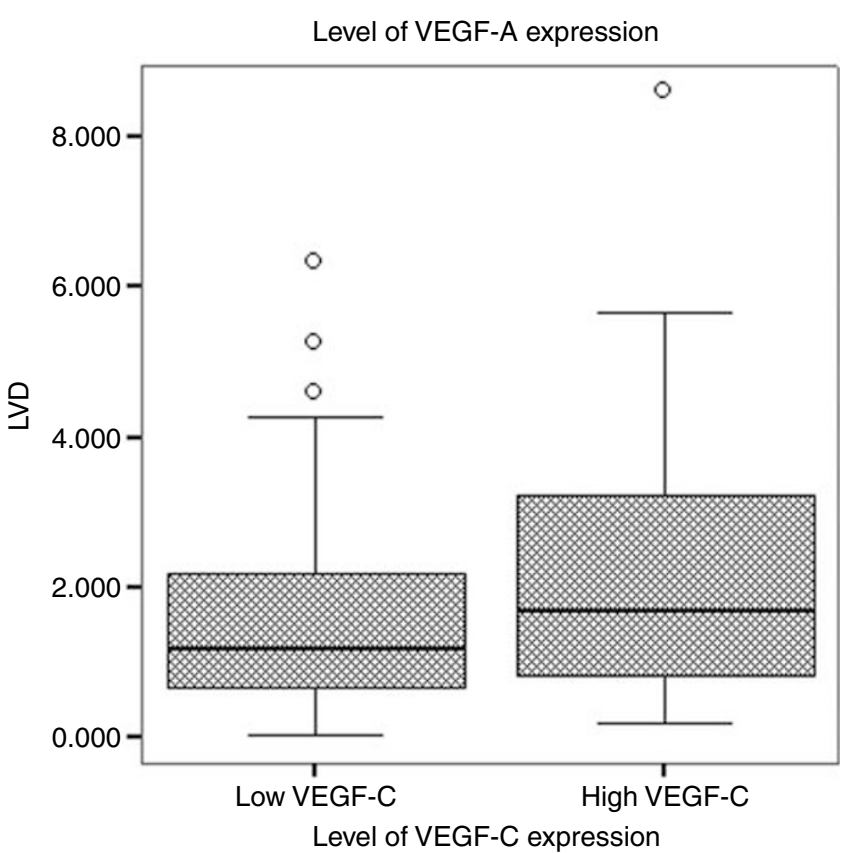

Figure 3 Box plots showing relationships between expression of VEGF-A and -C with MVD and LVD, with the middle line in each box representing the median value.

larger metastatic deposits, likely through the growth factor inducing a rich vascular network, and a correspondingly more nutritious environment for tumour growth. The current study also found that such tumours behaved more aggressively, as they were significantly associated with the presence of LN metastasis $(P<0.001)$, distant metastasis $(P=0.01)$ and a poorer survival $(P=0.029)$. These findings are similar to others, both in breast cancer and other tumour types (Toi et al, 1995b; Linderholm et al, 1999; O-Charoenrat et al, 2001). Although the effect of VEGF-A expression can be mediated by an increased vascular network, it should be noted that the growth factor has also been shown to increase breast cancer cell survival through direct action on VEGFR-2 that has been found to be expressed on the surface of the breast cancer cells. Vascular endothelial cell growth factor-A production by, and VEGFR-2 activation on, the surface of tumour cells indicates the presence of a distinct autocrine signalling loop that enables breast cancer cells to promote their own growth and survival by activation of VEGFR-2 (Weigand et al, 2005). The findings in this study support others that have reported the important role of VEGF-A in the progression of breast cancer; a role mediated through angiogenesis and promotion of tumour cell survival, and add the induction of lymphangiogenesis as another possible mechanism.

Similar to previous reports (Gunningham et al, 2000), the present study detected positive staining for VEGF-C and -D in normal mammary epithelial cells at a weaker intensity than in the surrounding invasive component suggesting a role in remodelling lymphatic and blood vasculature in mammary stroma during the menstrual cycle. It is known that the normal human mammary gland undergoes a well-defined sequence of changes in both epithelial and stromal compartments during the menstrual cycle, with increased angiogenesis during the ovulatory phase under effect of hormonal changes (Ferguson et al, 1992; Weinstein et al, 2005). 

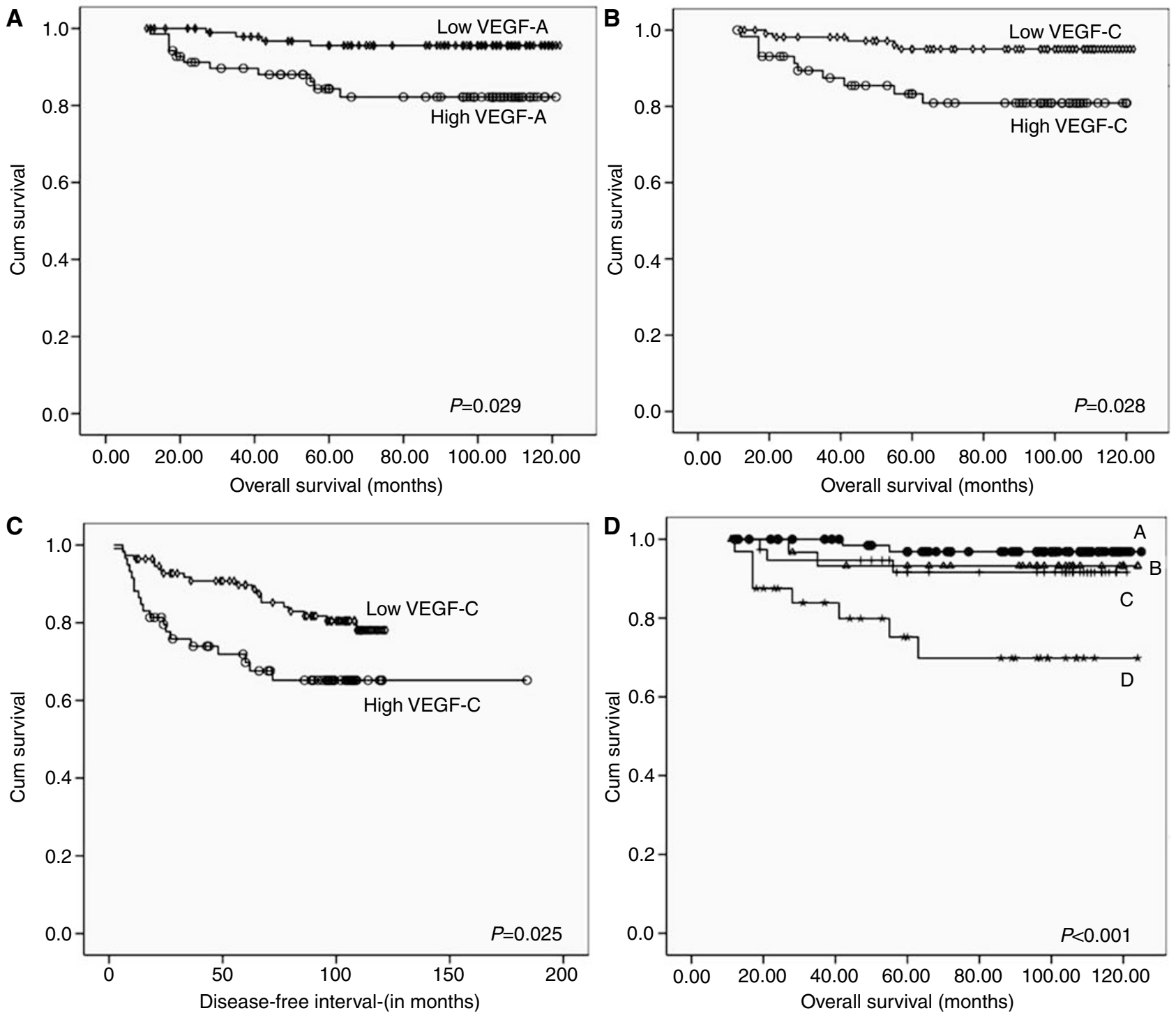

Figure 4 Associations between expression of VEGF-A and $-C$ with patient prognosis using Kaplan-Meier method. High expression of VEGF-A is significantly associated with decreased OS (A). High expression of VEGF-C is significantly associated with decreased OS (B) and DFI (C). Comparison between survival in four groups: group A (low expression of both VEGF-A and VEGF-C, group B; (high expression VEGF-A ), group C; (high expression of VEGF-C) and group D; (high expression of both VEGF-A and VEGF-C) showing that group D has the worst prognosis.

Using animal models, VEGF-C induced formation of new lymph vessels in the chick chorioallantoic membrane (Oh et al, 1997), induced lymphangiogenesis around islet of Langerhans in the pancreas which, normally, are not surrounded with lymphatics (Mandriota et al, 2001), induced hyperplasia and dilatation of the dermal lymphatics when overexpressed in transgenic mice (Jeltsch et al, 1997) and increased LVD and LN metastasis in xenografted breast cancer (Skobe et al, 2001). The present study reports a significant association between expression of VEGF-C and LVD similar to other findings reported in breast cancer (Nakamura et al, 2005) and other tumour types (Yonemura et al, 1999; Onogawa et al, 2004). This is mostly likely due to the stimulatory effect of VEGF-C on VEGFR-3 and $\alpha 9 \quad \beta 1$ integrin on the LEC surface. Stimulation of VEGFR-3 transmits potent mitogenic signals into LECs, whereas activation of integrin induces redistribution of the cellular actin cytoskeleton, leading to change in cell shape and formation of pseudopodia enabling cells to migrate eventually new lymphangiogenesis.

When processed through proteolysis in the ECM to mature forms, VEGF-C has been reported to also activate VEGFR-2 on the surface of the BECs, thereby inducing angiogenesis (Joukov et al, 1996). Such effects have been reported in experimental studies (Oh et al, 1997), but has not been fully studied in human breast cancer. Unlike a recent report by $\mathrm{Hu}$ et al (2005), the present study indicates that tumours with high expression of VEGF-C are significantly associated with a higher MVD, suggesting that VEGF-C has an additional angiogenic effect in breast cancer and may reflect active proteolysis found in the tumour microenvironment. The study by $\mathrm{Hu}$ and co-workers found no such association, however, such differences may reflect the different patient population and the smaller number of specimens in the study by $\mathrm{Hu}$.

Similar to other studies (Nakamura et al, 2003b), when high expression of VEGF-C was examined in relation to the clinicopathological characteristics and to patient survival, a significant positive relationship was found with the presence of both LN metastasis $(P<0.001)$ and distant metastasis $(P=0.008)$. High expression was also significantly associated with the occurrence of regional recurrence $(P=0.025)$, and poorer OS $(P=0.028)$, perhaps due to the potent ability of VEGF-C to induce both 
lymphangiogenesis and angiogenesis, thereby providing open channels for dissemination of malignant cells.

Tumours which express high levels of both VEGF-A and -C were found to have the worst prognosis when compared with tumours expressing low levels of both factors or tumours expressing high levels of either of the growth factors individually. These results support the notion that the two VEGF family members may be involved in tumour progression at two interrelated steps. Evaluation of expression of both VEGF-A and -C may be an important factor to identify breast cancer patients at higher risk of recurrence and in need of adjuvant therapy.

VEGF-D is a relatively recent member of the VEGF family that shares some structural and functional properties with VEGF-C (Yamada et al, 1997). In experimental animal tumour models, overexpression of VEGF-D was found to be significantly associated with higher rate of LN metastasis, increased tumour angiogenesis and larger tumour sizes (Stacker et al, 2001; Von Marschall et al, 2005; Yonemura et al, 2005). However, in human tumours, there is variation amongst results. It has been reported to be significantly associated with high LVD, LN metastasis and poorer survival in gastric (Shida et al, 2005), endometrial (Yokoyama et al, 2003) and colorectal carcinomas (White et al, 2002). In head and neck tumours (O-Charoenrat et al, 2001), such relationships have not been found. In other studies in breast and in lung carcinoma, low levels of expression of VEGF-D was associated with poorer prognosis (Niki et al, 2000; Koyama et al, 2003). In the current study, there was a trend for tumours with higher VEGF-D expression to have a higher rate of $\mathrm{LN}$ metastasis, regional recurrence and distant metastasis, however, none of these reached statistical significance. Similar findings were reported by Yang et al (2002). In addition, no significant association was found between

\section{REFERENCES}

Bevilacqua P, Barbareschi M, Verderio P, Boracchi P, Caffo O, Dalla Palma P, Meli S, Weidner N, Gasparini G (1995) Prognostic value of intratumoral microvessel density, a measure of tumor angiogenesis, in node-negative breast carcinoma - results of a multiparametric study. Breast Cancer Res Treat 36: 205-217

Brown LF, Berse B, Jackman RW, Tognazzi K, Manseau EJ, Dvorak HF, Senger DR (1993) Increased expression of vascular permeability factor (vascular endothelial growth factor) and its receptors in kidney and bladder carcinomas. Am J Pathol 143: 1255-1262

Choi WW, Lewis MM, Lawson D, Yin-Goen Q, Birdsong GG, Cotsonis GA, Cohen C, Young AN (2005) Angiogenic and lymphangiogenic microvessel density in breast carcinoma: correlation with clinicopathologic parameters and VEGF-family gene expression. Mod Pathol 18: $143-152$

Currie MJ, Hanrahan V, Gunningham SP, Morrin HR, Frampton C, Han C, Robinson BA, Fox SB (2004) Expression of vascular endothelial growth factor D is associated with hypoxia inducible factor (HIF-1alpha) and the HIF-1alpha target gene DEC1, but not lymph node metastasis in primary human breast carcinomas. J Clin Pathol 57: 829-834

Des Guetz G, Uzzan B, Nicolas P, Cucherat M, Morere JF, Benamouzig R, Breau JL, Perret GY (2006) Microvessel density and VEGF expression are prognostic factors in colorectal cancer. Meta-analysis of the literature. $\mathrm{Br}$ J Cancer 94: 1823-1832

Ferguson JE, Schor AM, Howell A, Ferguson MW (1992) Changes in the extracellular matrix of the normal human breast during the menstrual cycle. Cell Tissue Res 268: 167-177

Ferrara N, Bunting S (1996) Vascular endothelial growth factor, a specific regulator of angiogenesis. Curr Opin Nephrol Hypertens 5: 35-44

Ferrara N, Davis-Smyth T (1997) The biology of vascular endothelial growth factor. Endocr Rev 18: 4-25

Fox SB, Harris AL (2004) Histological quantitation of tumour angiogenesis. Apmis 112: 413-430

Fox SB, Gasparini G, Harris AL (2001) Angiogenesis: pathological, prognostic, and growth-factor pathways and their link to trial design and anticancer drugs. Lancet Oncol 2: 278-289 expression of VEGF-D and either LVD or MVD. In a study on breast cancer, a significant association was found between high VEGF-D gene expression, using real-time polymerase chain reaction and LVD (Choi et al, 2005). Such differences in results may be due to the different methods in assessment of VEGF-D expression. Although VEGF-D showed lymphangiogenic and angiogenic effects in xenografted tumours, its biological role in human breast cancer is still unclear and is in need of further study. It is probable that VEGF-D plays different roles in different tumour types.

In conclusion, it appears from this study of human breast cancers that, as has been reported by in vitro studies, VEGF-A plays a role in lymphangiogenesis. It also appears that breast cancers, which express high levels of VEGF-A and -C are characterised by greater angiogenesis and lymphangiogenesis and are associated with the presence of both LN and distant metastasis. Such tumours behave more aggressively as indicated by the associations with shorter DFI and OS. Both growth factors appear to play an important role in the progression of breast carcinoma and to have a significant impact on patient prognosis. Examination of expression of either VEGF-A and -C can be used to identify a subset of breast cancer at higher risk for development of recurrence and distant metastasis, with the recommendation that both be assessed to further delineate those at highest risk.

\section{ACKNOWLEDGEMENTS}

We thank Mrs Kelly Huber and Ms Aula Ammar for their kind technical help.
Fuckar D, Dekanic A, Stifter S, Mustac E, Krstulja M, Dobrila F, Jonjic N (2006) VEGF expression is associated with negative estrogen receptor status in patients with breast cancer. Int J Surg Pathol 14: 49-55

Giorgadze TA, Zhang PJ, Pasha T, Coogan PS, Acs G, Elder DE, Xu X (2004) Lymphatic vessel density is significantly increased in melanoma. J Cutan Pathol 31: 672-677

Gunningham SP, Currie MJ, Han C, Robinson BA, Scott PA, Harris AL, Fox SB (2000) The short form of the alternatively spliced flt- 4 but not its ligand vascular endothelial growth factor $\mathrm{C}$ is related to lymph node metastasis in human breast cancers. Clin Cancer Res 6: 4278-4286

Han H, Silverman JF, Santucci TS, Macherey RS, d'Amato TA, Tung MY, Weyant RJ, Landreneau RJ (2001) Vascular endothelial growth factor expression in stage I non-small cell lung cancer correlates with neoangiogenesis and a poor prognosis. Ann Surg Oncol 8: 72-79

Hicklin DJ, Ellis LM (2005) Role of the vascular endothelial growth factor pathway in tumor growth and angiogenesis. J Clin Oncol 23: $1011-1027$

Hong YK, Lange-Asschenfeldt B, Velasco P, Hirakawa S, Kunstfeld R, Brown LF, Bohlen P, Senger DR, Detmar M (2004) VEGF-A promotes tissue repair-associated lymphatic vessel formation via VEGFR-2 and the alphalbetal and alpha2betal integrins. FASEB J 18: $1111-1113$

Hu SE, Zhang YJ, Cui YM, Zhang HQ (2005) Expression of vascular endothelial growth factor $\mathrm{A}$ and $\mathrm{C}$ in human breast cancer and their significance. Ai Zheng 24: 1076-1079

Jeltsch M, Kaipainen A, Joukov V, Meng X, Lakso M, Rauvala H, Swartz M, Fukumura D, Jain RK, Alitalo K (1997) Hyperplasia of lymphatic vessels in VEGF-C transgenic mice. Science 276: 1423-1425

Jennbacken K, Vallbo C, Wang W, Damber JE (2005) Expression of vascular endothelial growth factor C (VEGF-C) and VEGF receptor-3 in human prostate cancer is associated with regional lymph node metastasis. Prostate 65: $110-116$

Joukov V, Pajusola K, Kaipainen A, Chilov D, Lahtinen I, Kukk E, Saksela O, Kalkkinen N, Alitalo K (1996) A novel vascular endothelial growth factor, VEGF-C, is a ligand for the Flt4 (VEGFR-3) and KDR (VEGFR-2) receptor tyrosine kinases. EMBO J 15: 1751 
Kadowaki I, Ichinohasama R, Harigae H, Ishizawa K, Okitsu Y, Kameoka J, Sasaki T (2005) Accelerated lymphangiogenesis in malignant lymphoma: possible role of VEGF-A and VEGF-C. Br J Haematol 130: 869-877

Kleespies A, Bruns CJ, Jauch KW (2005) Clinical significance of VEGF-A, -C and -D expression in esophageal malignancies. Onkologie 28: $281-288$

Koskinen WJ, Bono P, Leivo I, Vaheri A, Aaltonen LM, Joensuu H (2005) Lymphatic vessel density in vocal cord carcinomas assessed with LYVE-1 receptor expression. Radiother Oncol 77: $172-175$

Koyama Y, Kaneko K, Akazawa K, Kanbayashi C, Kanda T, Hatakeyama K (2003) Vascular endothelial growth factor-C and vascular endothelial growth factor-d messenger RNA expression in breast cancer: association with lymph node metastasis. Clin Breast Cancer 4: 354-360

Kunstfeld R, Hirakawa S, Hong YK, Schacht V, Lange-Asschenfeldt B, Velasco P, Lin C, Fiebiger E, Wei X, Wu Y, Hicklin D, Bohlen P, Detmar $M$ (2004) Induction of cutaneous delayed-type hypersensitivity reactions in VEGF-A transgenic mice results in chronic skin inflammation associated with persistent lymphatic hyperplasia. Blood 104: 1048-1057

Linderholm B, Tavelin B, Grankvist K, Henriksson R (1999) Does vascular endothelial growth factor (VEGF) predict local relapse and survival in radiotherapy-treated node-negative breast cancer? $\mathrm{Br} J$ Cancer 81: $727-732$

Mandriota SJ, Jussila L, Jeltsch M, Compagni A, Baetens D, Prevo R, Banerji S, Huarte J, Montesano R, Jackson DG, Orci L, Alitalo K, Christofori G, Pepper MS (2001) Vascular endothelial growth factor-C-mediated lymphangiogenesis promotes tumour metastasis. EMBO J 20: 672-682

Nagy JA, Vasile E, Feng D, Sundberg C, Brown LF, Detmar MJ, Lawitts JA, Benjamin L, Tan X, Manseau EJ, Dvorak AM, Dvorak HF (2002) Vascular permeability factor/vascular endothelial growth factor induces lymphangiogenesis as well as angiogenesis. J Exp Med 196: 1497-1506

Nakamura Y, Yasuoka H, Tsujimoto M, Imabun S, Nakahara M, Nakao K, Nakamura M, Mori I, Kakudo K (2005) Lymph vessel density correlates with nodal status, VEGF-C expression, and prognosis in breast cancer. Breast Cancer Res Treat 91: 125-132

Nakamura Y, Yasuoka H, Tsujimoto M, Yang Q, Imabun S, Nakahara M, Nakao K, Nakamura M, Mori I, Kakudo K (2003a) Prognostic significance of vascular endothelial growth factor $\mathrm{D}$ in breast carcinoma with long-term follow-up. Clin Cancer Res 9: 716-721

Nakamura Y, Yasuoka H, Tsujimoto M, Yang Q, Tsukiyama A, Imabun S, Nakahara M, Nakao K, Nakamura M, Mori I, Kakudo K (2003b) Clinicopathological significance of vascular endothelial growth factor-C in breast carcinoma with long-term follow-up. Mod Pathol 16: 309-314

Niki T, Iba S, Tokunou M, Yamada T, Matsuno Y, Hirohashi S (2000) Expression of vascular endothelial growth factors A, B, C, and D and their relationships to lymph node status in lung adenocarcinoma. Clin Cancer Res 6: $2431-2439$

O-Charoenrat P, Rhys-Evans P, Eccles SA (2001) Expression of vascular endothelial growth factor family members in head and neck squamous cell carcinoma correlates with lymph node metastasis. Cancer 92: $556-568$

Oh SJ, Jeltsch MM, Birkenhager R, McCarthy JE, Weich HA, Christ B, Alitalo K, Wilting J (1997) VEGF and VEGF-C: specific induction of angiogenesis and lymphangiogenesis in the differentiated avian chorioallantoic membrane. Dev Biol 188: 96-109

Onogawa S, Kitadai Y, Tanaka S, Kuwai T, Kimura S, Chayama K (2004) Expression of VEGF-C and VEGF-D at the invasive edge correlates with lymph node metastasis and prognosis of patients with colorectal carcinoma. Cancer Sci 95: 32 - 39

Partanen TA, Paavonen K (2001) Lymphatic versus blood vascular endothelial growth factors and receptors in humans. Microsc Res Tech 55: $108-121$

Rodriguez-Niedenfuhr M, Papoutsi M, Christ B, Nicolaides KH, von Kaisenberg CS, Tomarev SI, Wilting J (2001) Proxl is a marker of ectodermal placodes, endodermal compartments, lymphatic endothelium and lymphangioblasts. Anat Embryol (Berlin) 204: 399-406

Sahni D, Robson A, Orchard G, Szydlo R, Evans AV, Russell-Jones R (2005) The use of LYVE-1 antibody for detecting lymphatic involvement in patients with malignant melanoma of known sentinel node status. J Clin Pathol 58: $715-721$
Schacht V, Dadras SS, Johnson LA, Jackson DG, Hong YK, Detmar M (2005) Up-regulation of the lymphatic marker podoplanin, a mucin-type transmembrane glycoprotein, in human squamous cell carcinomas and germ cell tumors. Am J Pathol 166: 913 -921

Shida A, Fujioka S, Ishibashi Y, Kobayashi K, Nimura H, Mitsumori N, Suzuki Y, Kawakami M, Urashima M, Yanaga K (2005) Prognostic significance of vascular endothelial growth factor $\mathrm{D}$ in gastric carcinoma. World J Surg 29: 1600 - 1607

Skobe M, Hawighorst T, Jackson DG, Prevo R, Janes L, Velasco P, Riccardi L, Alitalo K, Claffey K, Detmar M (2001) Induction of tumor lymphangiogenesis by VEGF-C promotes breast cancer metastasis. Nat Med 7: $192-198$

Stacker SA, Caesar C, Baldwin ME, Thornton GE, Williams RA, Prevo R, Jackson DG, Nishikawa S, Kubo H, Achen MG (2001) VEGF-D promotes the metastatic spread of tumor cells via the lymphatics. Nat Med 7: 186-191

Toi M, Inada K, Hoshina S, Suzuki H, Kondo S, Tominaga T (1995a) Vascular endothelial growth factor and platelet-derived endothelial cell growth factor are frequently coexpressed in highly vascularized human breast cancer. Clin Cancer Res 1: $961-964$

Toi M, Inada K, Suzuki H, Tominaga T (1995b) Tumor angiogenesis in breast cancer: its importance as a prognostic indicator and the association with vascular endothelial growth factor expression. Breast Cancer Res Treat 36: 193-204

Von Marschall Z, Scholz A, Stacker SA, Achen MG, Jackson DG, Alves F, Schirner M, Haberey M, Thierauch KH, Wiedenmann B, Rosewicz S (2005) Vascular endothelial growth factor-D induces lymphangiogenesis and lymphatic metastasis in models of ductal pancreatic cancer. Int Oncol 27: $669-679$

Weidner N, Folkman J, Pozza F, Bevilacqua P, Allred EN, Moore DH, Meli S, Gasparini G (1992) Tumor angiogenesis: a new significant and independent prognostic indicator in early-stage breast carcinoma. J Natl Cancer Inst 84: 1875-1887

Weidner N, Semple JP, Welch WR, Folkman J (1991) Tumor angiogenesis and metastasis - correlation in invasive breast carcinoma. $N$ Engl J Med 324: $1-8$

Weigand M, Hantel P, Kreienberg R, Waltenberger J (2005) Autocrine vascular endothelial growth factor signalling in breast cancer. Evidence from cell lines and primary breast cancer cultures in vitro. Angiogenesis 8: $197-204$

Weinstein SP, Conant EF, Sehgal CM, Woo IP, Patton JA (2005) Hormona variations in the vascularity of breast tissue. J Ultrasound Med 24: 67-72; quiz 74

White JD, Hewett PW, Kosuge D, McCulloch T, Enholm BC, Carmichael J, Murray JC (2002) Vascular endothelial growth factor-D expression is an independent prognostic marker for survival in colorectal carcinoma. Cancer Res 62: $1669-1675$

Yamada Y, Nezu J, Shimane M, Hirata Y (1997) Molecular cloning of a novel vascular endothelial growth factor, VEGF-D. Genomics 42: $483-488$

Yang W, Klos K, Yang Y, Smith TL, Shi D, Yu D (2002) ErbB2 overexpression correlates with increased expression of vascular endothelial growth factors A, C, and D in human breast carcinoma. Cancer 94: $2855-2861$

Yokoyama Y, Charnock-Jones DS, Licence D, Yanaihara A, Hastings JM, Holland CM, Emoto M, Sakamoto A, Sakamoto T, Maruyama H, Sato S, Mizunuma H, Smith SK (2003) Expression of vascular endothelial growth factor (VEGF)-D and its receptor, VEGF receptor 3 , as a prognostic factor in endometrial carcinoma. Clin Cancer Res 9: $1361-1369$

Yonemura Y, Endo Y, Fujita H, Fushida S, Ninomiya I, Bandou E, Taniguchi K, Miwa K, Ohoyama S, Sugiyama K, Sasaki T (1999) Role of vascular endothelial growth factor $\mathrm{C}$ expression in the development of lymph node metastasis in gastric cancer. Clin Cancer Res 5: $1823-1829$

Yonemura Y, Endo Y, Tabata K, Kawamura T, Yun HY, Bandou E, Sasaki T, Miura M (2005) Role of VEGF-C and VEGF-D in lymphangiogenesis in gastric cancer. Int J Clin Oncol 10: 318-327 